Nuclear Physics B (Proc. Suppl.) XXX (2018) 1-3

North-Holland

\title{
PATH INTEGRALS AND VORONIN'S THEOREM ON THE UNIVERSALITY OF THE RIEMANN ZETA FUNCTION
}

\author{
Khalil M. Bitar \\ Supercomputer Computations Research Institute, Florida State University, Tallahassee, FL, USA 32306-3006
}

We explore a new approach to the path integral for a latticized quantum theory.

This talk is based on work with N. Khuri and H. Ren.[1.2]

The main new tools we use are theorems by Voronin on the universality properties of the Riemann Zeta function, $\zeta(s)$, in the critical strip, $\frac{1}{2}<\operatorname{Re} s<1$.

Given any real continuous function, $\phi(x), 0 \leq$ $x \leq \mathrm{E}$, we can choose a mapping, $s(x)$, which maps the line, $0 \leq x \leq \mathrm{E}$, onto a line $s(x)$ that lies in the strip $\frac{1}{2}<\operatorname{Re} s<1$, then given any $\Delta>0$, we have an infinite set of integers, $\mathcal{L}$, such that for all $n \in \mathcal{L}$,

$$
\mid \phi(x)-\gamma(s(x)+\text { in } \Delta) \mid<\varepsilon, \quad 0 \leq x \leq \mathrm{E}
$$

here $s(x)$ is fixed and could be taken to be linear, $s(x)=\frac{1}{2}+\frac{x}{2 \mathrm{E}}$; and $\epsilon$ is arbitrarily small, $\gamma=$ $\ln |\zeta|$.

Thus for all integers $n$ the functions $\left\{\gamma\left(s\left(x_{j}\right)\right.\right.$ + in $\Delta)\}$ will come arbitrarily close to "all paths" $\left\{\phi\left(x_{j}\right)\right\}, 0 \leq x, \leq \mathrm{E}, \quad j=1,2, \ldots, \nu$.

The path integral may be written as a discrete sum over paths labeled by an integer, $n$, and given by $\left\{\gamma_{i}\left(s\left(x_{j}\right)+i n \Delta\right)\right\}, j=1, \ldots, \nu$.

One then has to introduce a measure, or density function, $\rho_{\nu}(n)$, which essentially counts the number of different integers " $n_{\ell}$ " which give paths equivalent to $n$.

\section{Path Integrals}

In quantum mechanics or quantum field theory one has to evaluate integrals of the following from:

$$
\begin{aligned}
\langle\mathrm{P}(\phi)\rangle= & \frac{1}{\Omega} \int \prod_{j=1}^{\nu} d \phi(j) e^{-\mathrm{S}(\phi(j))} \\
& \times \mathrm{P}\left(\phi\left(\ell_{1}\right) \ldots \phi\left(\ell_{m}\right)\right)
\end{aligned}
$$

with

$$
\Omega \quad=\int \prod_{j=1}^{\nu} d \phi(j) e^{-\mathrm{S}(\phi(j))}
$$

Here $\nu$ is the number of lattice points, $\phi(j)$ is the field at the $j$ 'th lattice point, $\mathrm{S}(\phi)$ is the Euclidean action, and $\mathrm{P}\left(\phi\left(\ell_{1}\right) \ldots \phi\left(\ell_{m}\right)\right)$ is a polynomial in the fields.

Such an integral can be easily well approximated by a summation.

We shall assume that $\rho_{\nu}$ exits and is a smooth function of $\gamma$.

\section{Properties of the Measure $\rho(\mathbf{n})$}

We concentrate here on the central question of this talk, namely the properties of the "density" or "measure", $\rho_{\nu}(n)$. Indeed we shall derive an 
explicit integral representation for it.

The first thing to do is to actually plot the distribution of values of zeta functions. We consider the set of zeta functions: $\zeta\left(\sigma+i n \Delta+i \mathrm{~T}_{0}\right) ; n=$ $1,2, \ldots, \mathrm{N} ; \frac{1}{2}<\sigma<1$; any real $\Delta>0$ (actually $\Delta>10$, see below), and a fixed $\mathrm{T}_{0}>O\left(10^{6}\right)$ and compute their values.

This computation strongly indicates that there exists a probability distribution, $\mathrm{W}_{\sigma}(\gamma)$ independent of $\Delta$ and of $\mathrm{T}_{0}$ (for large $\mathrm{T}_{0}$ ), which tells us that if we randomly choose a $t>\mathrm{T}_{0}$ and calculate $\gamma(\sigma+i t)$, the probability that $u<\gamma<u+d u$, is given by $\mathrm{W}_{\sigma}(u) d u$.

We define our $n$ 'th "path" by the configuration

$$
\begin{gathered}
\phi^{(n)}\left(x_{j}\right) \equiv \log |\zeta(\sigma+i j h+i n \Delta)|=\gamma_{\sigma}(j, n) \\
j=1, \ldots, \nu .
\end{gathered}
$$

Here to separate our paths we take $\Delta \gg h$, and $\Delta>h \nu$.

It now follows from the standard definition of the path integral that one can write

$\Omega(\nu)=\sum_{n=N_{0}}^{N} \frac{e^{-S(n ; \nu)}}{\rho_{\nu}(n)}+\mathcal{O}\left(N^{-\frac{1}{\nu}}\right)$.

We must take $N$ large, and $N \gg N_{0}$. By summing over $n$ we sum over all "paths", but the density $\rho_{\nu}(n)$ insures that we have the correct Jacobian for quantum mechanics.

The main question here is to find the expression for, $\rho_{\nu}(n)$.

We first discuss the case $\nu=1$. Here $\rho_{1}(n)$ can be computed once we know the asymptotic probability for having $|\zeta(\sigma+i t)|$, for a randomly chosen $t \gg 1$, be such that $r_{1}<|\zeta(\sigma+i t)|<r_{2}$. We call this probability density, $\mathcal{P}_{\sigma}(r)$, and write

$$
\begin{aligned}
\text { Prob. }\left(r_{1}<|\zeta(\sigma+i t)|<r_{2}\right) \equiv & \int_{r_{1}}^{r_{2}} \mathcal{P}_{\sigma}(r) d r \\
& \frac{1}{2}<\sigma<1 .
\end{aligned}
$$

The moments of $\mathcal{P}_{\sigma}(r)$ are known for $0 \leq$ Re $k \leq 2$,

$$
\begin{aligned}
\int_{0}^{\infty} \mathcal{P}_{\sigma}(r) r^{2 k} d r \equiv & \lim _{T \rightarrow \infty} \frac{1}{T} \int_{1}^{\infty}|\zeta(\sigma+i t)|^{2 k} d t \\
& =F_{k}(2 \sigma)
\end{aligned}
$$

and

$F_{k}(2 \sigma)=\prod_{p}{ }_{2} F_{1}\left(k, k, 1, \frac{1}{p^{2 \sigma}}\right)$

where ${ }_{2} F_{1}(a, b ; c ; z)$ is the standard hypergeometric function, and $\Pi_{p}$ is a product over all primes. We can obtain an explicit expression for $\mathcal{P}_{\sigma}(r)$ by taking the inverse Mellin transform and get

$\mathcal{P}_{\sigma}(r)=r^{-c} \int_{-\infty}^{+\infty} d \lambda(r)^{-i \lambda} F_{\frac{i \lambda}{2}+\frac{(c-1)}{2}}(2 \sigma)$

where we can take any real $\mathrm{c}$ such that $1<c<5$. The integral above is absolutely convergent. It is now easy to obtain the distribution function of the values of $\log |\zeta|$, which we call $W_{\sigma}(\gamma)$,

$\operatorname{Prob} .\left(\gamma_{1}<|\zeta(\sigma+i t)|<\gamma_{2}\right) \equiv \int_{\gamma_{1}}^{\gamma_{2}} W_{\sigma}(\gamma) d \gamma($

for a randomly chosen $t \gg 1$. It is clear that $W_{\sigma}(\gamma) \equiv \mathcal{P}_{\sigma}\left(e^{\gamma}\right) \cdot e^{\gamma}$, and hence

$$
\begin{aligned}
& W_{\sigma}(\gamma)=e^{-(c-1) \gamma} \int_{-\infty}^{+\infty} \\
& d \lambda e^{-i \lambda \gamma} F_{\frac{i \lambda}{2}+\frac{(c-1)}{2}}(2 \sigma)
\end{aligned}
$$

with $1<c<5$. The probability density $W_{\sigma}(\gamma)$ is an asymptotic distribution in the sense that if we take an interval $T_{0}<t<T, T \gg T_{0}$, and compute a large ensemble of $\log \left|\zeta\left(\sigma+i t_{j}\right)\right|$ values, then the resulting histogram for the distribution of values for this ensemble will approach $W_{\sigma}(\gamma)$ as $T \rightarrow \infty$, and $T_{0}$ is kept fixed. However, it is fortunate that even for an interval $T_{0}=\mathcal{O}\left(10^{6}\right)$ and $T=\mathcal{O}\left(10^{9}\right)$ the computed histogram and 


$$
+\mathcal{O}\left(N^{-\frac{1}{\nu}}\right)
$$

For results of numerical tests, we refer to References [1] and [2].

\section{Conclusions and Remarks}

Fig. 1. Distribution of the values of $\gamma=\ln |\zeta|$ at $\sigma=$ 0.75 . The crosses denote points computed from the exact formula, and the line is the histogram from computing a large sample.

the exact result for $W_{\sigma}(\gamma)$ are quite close to each other. This fact is shown in the figure where the region $T_{0}<t<10^{9}$ which is easily accessible to computer is probed. The moments of $W_{\sigma}(\gamma)$ can be given exactly. [2]

\section{The Measure for Multiple Variables}

For $\nu=1$, the density $\rho_{1}(n)$ is simply given by $\rho_{1}(n)=W_{\sigma}(\gamma(1 ; n))$, with $\gamma(1 ; n)$ defined in eqn. (4). For $\nu>1$, and $h>1$, the values of $\gamma_{\sigma}(j ; n)$ and $\gamma_{\sigma}(j+l ; n)$ are uncorrelated. Thus the probability density is the product of the independent probabilities for each component. This leads to a factorization of $\rho_{\nu}(n)$ and we get

$\rho_{\nu}(n)=\prod_{j=1}^{\nu} W_{\sigma}\left(\gamma_{\sigma}(j ; n)\right)$

We now have, for example, the following explicit formulae for the partition function in Euclidean Quantum Mechanics:

$\Omega(\nu)=\sum_{n=1}^{N} e^{-S(n ; \nu)}\left[\prod_{j=1}^{\nu} W_{\sigma}\left(\gamma_{\sigma}(j ; n)\right)\right]^{-1}$
We end this paper with a miscellaneous set of remarks and give projections on further work.

(i) The calculations we have carried out demonstrate the validity of the expressions for the partition function in Euclidean quantum mechanics. This includes the validity of the factorization conjecture for the density $\rho_{\nu}(n)$.

(ii) The applications of our method could either be for actual numerical calculations or for obtaining formal results. It may also be used for a complex actions.

(iii) The continuous form of Voronin's theorem, leads us to contemplate a far reaching conjecture. This concerns taking the limit $a \rightarrow 0$, where $a$ is the lattice spacing. If in this limit a measure, $\rho_{\infty}(n)$, exists, then essentially any quantum mechanical problem can be reduced to quadratures.

The important thing to remember is that $\rho_{\infty}(n)$ depends only on the properties of the Riemann zeta function. All the physics enters through $S(n)$. Clearly, the existence of $\rho_{\infty}(n)$ and an explicit formula for it would be a remarkable achievement.

\section{References}

[1] Path Integrals and Voronin's Theorem on the Universality of the Riemann Zeta Function, K.M. Bitar, N.N. Khuri and H.C. Ren, Annals of Physics, 211, No. 1 172-196 (1991).

[2] Path Integrals and Discrete Sums, Khalil Bitar, N.N. Khuri and H.C. Ren, Physical Review Letters, 67 781-784 (1991). 